\title{
Extended $\mathrm{x}$-ray absorption fine structure study of phase transitions in the piezoelectric perovskite $\mathrm{K}_{0.5} \mathrm{Na}_{0.5} \mathrm{NbO}_{3}$
}

\author{
A. Kodre, ${ }^{1,2}$ J. Tellier, ${ }^{2, a)}$ I. Arčon, ${ }^{3,2}$ B. Malič, ${ }^{2}$ and M. Kosec ${ }^{2}$ \\ ${ }^{1}$ Faculty of Mathematics and Physics, University of Ljubljana, Jadranska 19, SI-1000 Ljubljana, Slovenia \\ ${ }^{2}$ Jožef Stefan Institute, Jamova 39, SI-1000 Ljubljana, Slovenia \\ ${ }^{3}$ University of Nova Gorica, Vipavska 13, SI-5000 Nova Gorica, Slovenia
}

(Received 27 February 2009; accepted 4 May 2009; published online 11 June 2009)

\begin{abstract}
Following an $\mathrm{x}$-ray diffraction study of phase transitions of the piezoelectric perovskite $\mathrm{K}_{0.5} \mathrm{Na}_{0.5} \mathrm{NbO}_{3}$ the structural changes of the material are studied using extended $\mathrm{x}$-ray absorption fine structure analysis, whereby the neighborhood of $\mathrm{Nb}$ atom is determined in the temperature range of monoclinic, tetragonal, and cubic phases. Within the entire range $\mathrm{Nb}$ atom is displaced from the center of the octahedron of its immediate oxygen neighbors, as witnessed by the splitting of $\mathrm{Nb}-\mathrm{O}$ distance. The model shows high prevalence of the displacement in the (111) crystallographic direction of the simple perovskite cell. The corresponding splitting of the $\mathrm{Nb}-\mathrm{Nb}$ distance is negligible. There is no observable disalignment of the linear $\mathrm{Nb}-\mathrm{O}-\mathrm{Nb}$ bonds from the ideal cubic arrangement, judging from the intensity of the focusing of the photoelectron wave on the $\mathrm{Nb}-\mathrm{Nb}$ scattering path by the interposed oxygen atom. As a general result, the phase transitions are found as an effect of the long-range order, while the placement of the atoms in the immediate vicinity remains largely unaffected. (C) 2009 American Institute of Physics.
\end{abstract}

[DOI: $10.1063 / 1.3143070]$

\section{INTRODUCTION}

Alkali niobates have been extensively studied for application in piezoelectric devices, supplanting the environmentally harmful $\mathrm{Pb}$-based complex perovskites ceramics. In the sodium potassium niobate $\left(\mathrm{K}_{x} \mathrm{Na}_{1-x} \mathrm{NbO}_{3}\right)$ system, the best dielectric and piezoelectric properties are found for the composition with $x=0.5,{ }^{1}$ close to the morphotropic phase boundary $x=0.475$ reported by Tennery and Hang. ${ }^{2}$ The information on its structure or symmetry, however, is scarce: in particular, the phase transitions of the material have not been studied from a structural point of view. Ahtee and Glazer ${ }^{3,4}$ described the octahedral tilting system in some $\mathrm{K}_{x} \mathrm{Na}_{1-x} \mathrm{NbO}_{3}$ perovskites with a low value of $x$ at different temperatures, in order to complete the phase diagram published by Jaffe et al. ${ }^{5}$ Yet the phase transition sequence of $\mathrm{K}_{0.5} \mathrm{Na}_{0.5} \mathrm{NbO}_{3}$ has not been described in these publications. The transition temperatures of some compositions have already been determined using electrical measurements by Egerton and Dillon. ${ }^{1}$ These temperatures have been confirmed by differential scanning calorimetry in our study, ${ }^{6}$ where we have shown that with increasing temperature, the samples in the range $x=[0.4-0.6]$ undergo first-order monoclinic-tetragonal and tetragonal-cubic transitions. The phase transitions of $\mathrm{K}_{0.5} \mathrm{Na}_{0.5} \mathrm{NbO}_{3}$ are found at 466.9 and at $681.9 \mathrm{~K}$, respectively.

In the x-ray diffraction (XRD) method, however, the positions of the atoms, especially the lighter ones, can be extracted only indirectly, and with a considerable effort. In extended x-ray absorption fine structure (EXAFS) analysis, on the other side, spatial relations between neighbor atoms are obtained directly. ${ }^{7}$ Since the useful electronic properties of

${ }^{a)}$ Electronic mail: jenny.tellier@ijs.si. the material stem mainly from the shift of the $\mathrm{Nb}$ atom from the central position in its octahedron of oxygen atoms, a $\mathrm{Nb}$ $\mathrm{K}$-edge EXAFS study of the structure of the material has been used to determine the immediate neighborhood of the $\mathrm{Nb}$ atom in the range of the phase transitions.

The same approach has been used by Lemeshko et al. ${ }^{8,9}$ to study alkali niobates in an extremely wide temperature range and for several values of $\mathrm{K} / \mathrm{Na}$ atomic ratio. The authors start from a hypothesized spherical shell of $\mathrm{Nb}$ atom loci and determine the distribution of actual positions in this model for the entire range of crystallographic phases of the material, limiting the analysis mostly to the first-neighbor shell.

In our study, the previous XRD analysis ${ }^{6}$ has been complemented with EXAFS. Nb K-edge spectra of the material have been recorded at three temperatures $(297,500$, and $750 \mathrm{~K}$ ), each chosen far enough from the transition points, with the crystal phase (monoclinic, tetragonal, or cubic) firmly established. We have started by examining the $\mathrm{Nb}$ displacement along three basic crystallographic directions. Extending the analysis to the second and third shell of atomic neighbors, we can draw some conclusions on the correlation of atomic positions beyond the immediate contact neighborhood.

\section{EXPERIMENTAL}

The synthesis of the $\mathrm{K}_{0.5} \mathrm{Na}_{0.5} \mathrm{NbO}_{3}$ composition was made by reaction of the two simple perovskites $\mathrm{KNbO}_{3}$ and $\mathrm{NaNbO}_{3}$ previously synthesized, mixed in stoichiometric ratio and annealed at $1100{ }^{\circ} \mathrm{C}$ during $4 \mathrm{~h}$. The procedure and XRD characterization of the obtained material is fully described in the previous paper. ${ }^{6}$ 


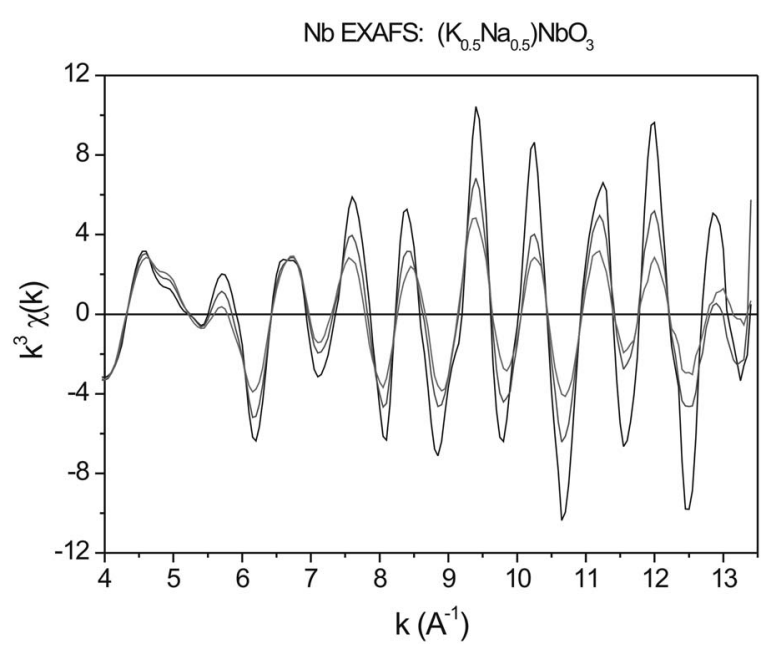

FIG. 1. The $k^{3}$ weighted EXAFS spectra of the $\mathrm{K}_{0.5} \mathrm{Na}_{0.5} \mathrm{NbO}_{3}$ sample measured at temperatures 297,500 , and $750 \mathrm{~K}$ in order of decreasing amplitude.

Absorption spectra were recorded at beamline $\mathrm{C}$ of HA$\mathrm{SYLAB}$, using a $\mathrm{Si}(111)$ double-crystal monochromator with about $2 \mathrm{eV}$ resolution at the $\mathrm{Nb} \mathrm{K}$-edge $(18986 \mathrm{eV})$. Higherorder harmonics were effectively eliminated by detuning of the monochromator crystals to $60 \%$ of the rocking curve maximum, using the beam-stabilization feedback control. The intensity of the x-ray beam was measured by three consecutive $10 \mathrm{~cm}$ long ionization detectors, filled with 1000 mbar $\mathrm{Ar}, 470$ mbar $\mathrm{Kr}$, and $900 \mathrm{mbar} \mathrm{Kr}$, respectively.

Powdered $\mathrm{K}_{0.5} \mathrm{Na}_{0.5} \mathrm{NbO}_{3}$ sample was mixed with micronized $\mathrm{BN}$ powder and pressed into homogeneous selfsupporting pellet with the total absorption thickness of about two above the $\mathrm{Nb} \mathrm{K}$-edge. The pellet was inserted between the first and the second ionization detector in a resistanceheated tubular oven of our own design, equipped with a programmable feedback controller and a direct reading of the sample temperature on-line with the absorption measurement. ${ }^{10,11}$

The absorption spectra were measured within the interval $(-250$ to $1000 \mathrm{eV})$ relative to the $\mathrm{Nb} \mathrm{K}$-edge. In the $\mathrm{X}$-ray absorption near edge structure (XANES) region equidistant energy steps of $0.5 \mathrm{eV}$ were used, while for the EXAFS region equidistant $k$-steps $\left(\Delta k \approx 0.05 \AA^{-1}\right)$ were adopted, with an integration time of $1 \mathrm{~s} / \mathrm{step}$. The exact energy calibration was established with a simultaneous absorption measurement on $\mathrm{Nb}$ metal foil placed between the second and the third ionization detector.

\section{RESULTS}

$\mathrm{Nb}$ EXAFS spectra (Fig. 1) were analyzed with IFEFFIT package $^{12}$ using FEFF6 code $^{13}$ in which the photoelectron scattering paths were calculated $a b$ initio from a presumed distribution of neighbor atoms. The Fourier transforms of the EXAFS spectra (Fig. 2) show contributions of photoelectron scattering on nearest neighbors around $\mathrm{Nb}$ in the crystal structure of $\mathrm{K}_{0.5} \mathrm{Na}_{0.5} \mathrm{NbO}_{3}$. The spectra show an overall decrease of EXAFS amplitude due to the increase of DebyeWaller factor with temperature. In order to obtain detailed quantitative structural data from the three temperature points, we modeled the spectra with an $a b$ initio FEFF model. Since

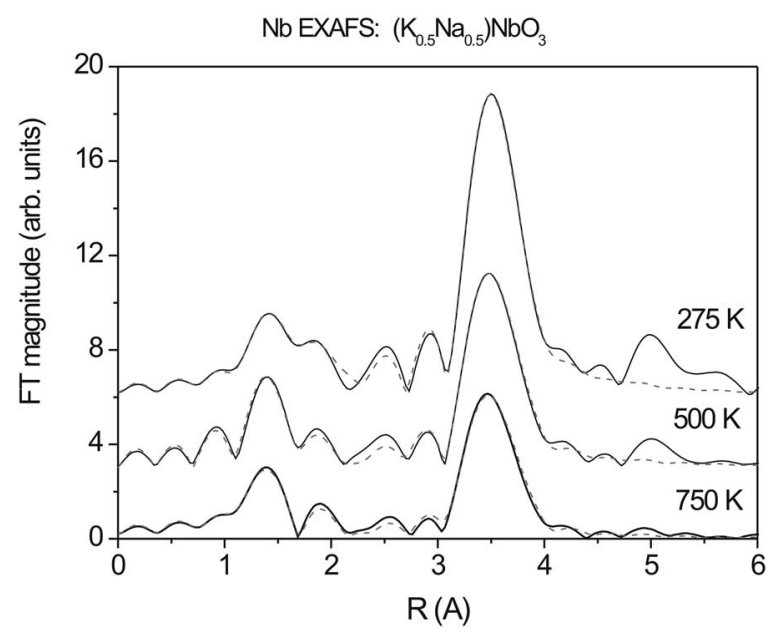

FIG. 2. Fourier transform magnitudes of the $k^{3}$ weighted EXAFS spectra from Fig. 1 together with best fit EXAFS model calculated in the $k$ range from 4 to $13 \AA^{-1}$ and $R$ range from 1.1 to $4.1 \AA$. The spectra are displaced vertically for clarity.

the deviations from the cubic phase are minute in any case, the basic model is built from crystallographic data for cubic phase with $P m-3 m$ space group $(a=4.002 \AA) .{ }^{6}$ In this crystal structure $\mathrm{Nb}$ atoms are located at the centers of oxygen octahedra with $\mathrm{Nb}-\mathrm{O}$ distance of $2.00 \AA$. The second coordination sphere at the distance of $3.47 \AA$ is occupied by, on average, four $\mathrm{K}$ and four $\mathrm{Na}$ atoms, randomly distributed over the equivalent crystallographic sites. The third $\mathrm{Nb}$ coordination shell is composed of six $\mathrm{Nb}$ atoms at $4.00 \AA$.

A qualitative comparison of the FEFF model with the Fourier transforms of measured spectra shows that a compound peak in the $R$ range between 1 and $2 \AA$ (Fig. 2) corresponds to oxygen neighbors, and a strong peak at about 3.5 $\AA$ to $\mathrm{Nb}$ neighbors. The amplitude of this peak is strongly enhanced by strong focusing of the photoelectron wave along the linear $\mathrm{Nb}-\mathrm{O}-\mathrm{Nb}$ configuration. The alkali atoms contribute a relatively weak signal at the foot of the $\mathrm{Nb}$ peak.

The relatively large width and bimodal shape of the firstneighbor peak in all three phases indicate a splitting of the nearest oxygen coordination shell. A deviation from the basic cubic structure is introduced artificially into the FEFF model by replacing the single sixfold degenerate $\mathrm{Nb}-\mathrm{O}$ scattering path with two or three paths with slightly different $\mathrm{Nb}-\mathrm{O}$ distances, to describe a small shift $\Delta R$ of $\mathrm{Nb}$ atom from the central position in the octahedron in one of the three basic directions in a simple perovskite cell:

(a) (111) direction resulting in $3+3$ splitting $(+\Delta R / \sqrt{ } 3$, $-\Delta R / \sqrt{3})$

(b) (110) direction resulting in $2+2+2$ splitting $(+\Delta R / \sqrt{ } 2,0,-\Delta R / \sqrt{ } 2)$

(c) (100) direction resulting in $1+4+1$ splitting $(+\Delta R, 0$, $-\Delta R)$

with the respective corrections to the $\mathrm{Nb}-\mathrm{O}$ distance given in the parentheses. The $\mathrm{Nb}-\mathrm{Nb}$ scattering paths from the linear $\mathrm{Nb}-\mathrm{O}-\mathrm{Nb}$ bonds are split accordingly, but with an adjustable correction parameter.

Each of these split models, together with the basic model 
TABLE I. Parameters of the nearest coordination shells around niobium atom in $\mathrm{K}_{0.5} \mathrm{Na}_{0.5} \mathrm{NbO}_{3}$ at three different temperatures: atomic species, coordination number $N$, distance $r$, and Debye-Waller factor $\sigma^{2}$. Statistical estimates provided by the FEFFIT package, of the uncertainty of the last digit are given in parentheses.

\begin{tabular}{|c|c|c|c|c|c|c|c|c|c|}
\hline \multirow[b]{2}{*}{ Neigh } & \multicolumn{3}{|c|}{$297 \mathrm{~K}$} & \multicolumn{3}{|c|}{$500 \mathrm{~K}$} & \multicolumn{3}{|c|}{$750 \mathrm{~K}$} \\
\hline & $N$ & $\begin{array}{c}r \\
(\AA)\end{array}$ & $\begin{array}{c}\sigma^{2} \\
\left(\AA^{2}\right)\end{array}$ & $N$ & $\begin{array}{c}r \\
(\AA)\end{array}$ & $\begin{array}{c}\sigma^{2} \\
\left(\AA^{2}\right)\end{array}$ & $N$ & $\begin{array}{c}r \\
(\AA)\end{array}$ & $\begin{array}{c}\sigma^{2} \\
\left(\AA^{2}\right)\end{array}$ \\
\hline $\mathrm{O}$ & $3.4(2)$ & $1.894(5)$ & $0.0039(2)$ & $3.6(2)$ & $1.914(5)$ & $0.0048(3)$ & $3.6(2)$ & $1.915(5)$ & $0.0055(4)$ \\
\hline $\mathrm{O}$ & $2.6(2)$ & $2.112(5)$ & $0.0039(2)$ & $2.4(2)$ & $2.115(5)$ & $0.0048(3)$ & $2.4(2)$ & $2.100(5)$ & $0.0055(4)$ \\
\hline K & 4 & $3.47(1)$ & $0.007(1)$ & 4 & $3.47(1)$ & $0.011(1)$ & 4 & $3.47(1)$ & $0.017(3)$ \\
\hline $\mathrm{Na}$ & 4 & $3.47(1)$ & $0.04(1)$ & 4 & $3.47(1)$ & $0.06(1)$ & 4 & $3.47(1)$ & $0.08(2)$ \\
\hline $\mathrm{Nb}$ & 6 & $4.00(1)$ & $0.0033(3)$ & 6 & $4.00(1)$ & $0.0053(3)$ & 6 & $4.00(1)$ & $0.0070(3)$ \\
\hline
\end{tabular}

with zero shift $\Delta R$, is used to fit the EXAFS spectra at three temperature points. The inclusion of the splitting improves the quality of the fit, given by the conventional measure of $R$-factor, for an order of magnitude over the basic model without splitting. The lowest $R$-factor, by a factor of 2 or more, is obtained in the case (a). The models with a combination of shift directions do not improve the fit but rather turn back to the case (a).

On the basis of the above analysis a more sophisticated FEFF model is constructed to refine the structural parameters by exploiting the redundancies in the data. In a simultaneous relaxation of the three EXAFS spectra from different temperature points, some parameters can be constrained to common values or to a monotonic variation with temperature. In particular, the Debye-Waller width parameters of a neighbor shell are supposed to follow the Einstein or Debye model of temperature dependence.

The model (a) comprises the consecutive neighbor shells described above, including all single-scattering paths up to 4 $\AA$, i.e., two $\mathrm{O}$ paths, the $\mathrm{K}$ and $\mathrm{Na}$ paths, and two $\mathrm{Nb}$ paths, together with three multiple-scattering focusing paths $\mathrm{Nb}$ $\mathrm{O}-\mathrm{Nb}$. The nine paths per each of three spectra require 22 parameters in all, among them three sets of Debye-Waller parameters and three sets of distance adjustments: the overall expansion coefficient, the $\mathrm{Nb}$ displacements $\Delta r$, and the corresponding splitting of $\mathrm{Nb}-\mathrm{Nb}$ paths. The stoichiometry is not varied: the degeneracy of individual scattering paths is left as defined from the crystallographic data.

The results of the relaxation procedure are given in Table I, and the quality of fits is graphically presented in Fig. 2. The $R$-factor of the simultaneous fit is close to the former values from separate fits, in spite of a reduction of the number of variable parameters per spectrum, so that the estimated error of the individual fitted parameters is effectively halved on average. The parameters $E_{0}$ for the shift of the photoelectron zero-energy point converge to a single value for the three spectra. Similarly, the values for the splitting of the $\mathrm{Nb}-\mathrm{Nb}$ distance converge to a common very small value, leading to the conclusion that the splitting of the $\mathrm{Nb}-\mathrm{Nb}$ scattering paths is superficial and can be removed. The Debye-Waller parameters for the $\mathrm{Nb}-\mathrm{Na}$ scattering tend to infinity suggesting that $\mathrm{Na}$, owing to its smaller mass and smaller ionic radius, occupies a much more fuzzy position within its cube space than $\mathrm{K}$. They regain a finite value only when constrained with a proportionality factor to the $\mathrm{Nb}-\mathrm{K}$ values. With these redundancies taken into account, there are in total 16 active parameters of the model against 49 independent points, obtained with the fitting ranges of 4-13 $\AA^{-1}$ in the $k$-space and from $1.1-4.1 \AA$ in $R$-space.

In an attempt to detect the (dis)alignment of $\mathrm{Nb}$ atoms, the scattering paths $\mathrm{Nb}-\mathrm{O}-\mathrm{Nb}$ describing the focusing of the photoelectron by the interposed $\mathrm{O}$ atoms are inspected in detail. The amplitude of the path, calculated by FEFF6 for the undeformed cubic lattice, shows maximum focusing effect, since the atoms are ideally aligned. Thus, any disalignment would result in a decrease in the amplitudes. When variable factors are introduced into the expression for the amplitudes, the values $1.01,1.00$, and 0.96 are obtained in the fit. The values are, within error bars, equal to unity, so that any systematic disalignment is precluded.

Another feature of the structure is modeled with parameter $q$, allowing for an adjustment of the split ratio, so that the amplitudes of the two oxygen paths are $3+q$ and $3-q$ rather than 3 and 3. The obtained values are 0.4 at $297 \mathrm{~K}$ and 0.6 at both high-temperature points, outside of the normal error interval of the occupation number parameters. Although only estimates, the values appear statistically significant: they point to a deformation of the oxygen octahedron coupled with the $\mathrm{Nb}$ displacement, rather than to a combination of displacement directions, which has been excluded by the initial numerical tests as described. The finding is probably connected with the observed change of the smaller $\mathrm{Nb}-\mathrm{O}$ distance from 1.894 to $1.914 \AA$, resolved reliably in the improved stability of the simultaneous fit. While the other interatomic distances stay constant with temperature, in accord with the fact that the effect of thermal expansion remains below detection limit, this change points to a deformation of the basic octahedron unit in the tetragonal-tomonoclinic phase transition. The steady increase of the Debye-Waller widths, on the other side, is most likely a direct effect of the temperature.

\section{CONCLUSION}

The simultaneous fitting of the EXAFS data at three temperature points improves the stability and accuracy of obtained parameters, so that a wider range of results is obtained. The basic findings, the size and direction of the $\mathrm{Nb}$ displacement, agree with the results of Lemeshko et al. ${ }^{8,9}$ In our results, additional information is obtained on the relative 
occupation of the split $\mathrm{O}$ subshells and on colinearity of the $\mathrm{Nb}-\mathrm{O}-\mathrm{Nb}$ bonds. Together with the absence of splitting in the $\mathrm{Nb}-\mathrm{Nb}$ distance, these data could provide a basis of a higher-level structural model of the material.

In summing up, it is evident that the displacement of the $\mathrm{Nb}$ atom does not correlate with the symmetry of the crystal lattice. It occurs randomly along one of the eight equivalent (111) directions of the simple perovskite cell, rather uncorrelated between adjacent octahedra since there is no splitting of the $\mathrm{Nb}-\mathrm{Nb}$ distances and no superlattice has been observed in XRD. The phase transitions do not affect the inside of the octahedra but rather their relative positions and orientations. A similar finding, a deviation from an ideal centrosymmetric structure, has been observed in the optical Raman scattering on the perovskite lattice, where a strong Raman line has persisted beyond the transition point well into the range of the cubic phase. ${ }^{14-16}$

\section{ACKNOWLEDGMENTS}

This work was supported by the Slovenian Research Agency research program P1-0112 and P2-0105, by DESY, and the European Community under the FP6 Programme "Structuring the European Research Area" contract No. RII3CT-2004-506008 (IA-SFS) and FP6 Network of Excellence MIND-Multifunctional \& Integrated Piezoelectric Devices (Grant No. NOE 515757-2). Access to synchrotron radiation facilities of HASYLAB (Project No. II-20080058 EC) is acknowledged. We would like to thank Edmund Welter of HASYLAB for expert advice on beamline operation.

${ }^{1}$ L. Egerton and D. M. Dillon, J. Am. Ceram. Soc. 42, 438 (1959).

${ }^{2}$ V. J. Tennery and K. W. Hang, J. Appl. Phys. 39, 4749 (1968).

${ }^{3}$ M. Ahtee and A. M. Glazer, Ferroelectrics 7, 93 (1974).

${ }^{4}$ M. Ahtee and A. M. Glazer, Acta Crystallogr. A 32, 434 (1976).

${ }^{5}$ B. Jaffe, W. Cook, and H. Jaffe, Piezoelectric Ceramics (Academic Press, London, New York, 1971).

${ }^{6}$ J. Tellier, B. Malic, B. Dkhil, D. Jenko, J. Cilensek, and M. Kosec, Solid State Sci. 11, 320 (2009).

${ }^{7}$ A. Kodre, I. Arčon, J. Padežnik Gomilšek, and B. Zalar, AIP Conf. Proc. 882, 481 (2007).

${ }^{8}$ M. P. Lemeshko, E. S. Nazarenko, A. A. Gonchar, L. A. Reznichenko, T. I. Nedoseykina, A. A. Novakovich, O. Mathon, Y. Joly, and R. V. Vedrinskii, Phys. Rev. B 76, 134106 (2007).

${ }^{9}$ M. P. Lemeshko, E. S. Nazarenko, A. A. Gonchar, L. A. Reznichenko, O. Mathon, Y. Joly, and R. V. Vedrinskii, Europhys. Lett. 77, 26003 (2007).

${ }^{10}$ R. Prešeren, I. Arčon, M. Mozetič, A. Kodre, and A. Pregelj, Nucl. Instrum. Methods Phys. Res. B 111, 161 (1996).

${ }^{11}$ A. Mihelič, A. Kodre, I. Arčon, J. Padežnik Gomilšek, and M. Borowski, Nucl. Instrum. Methods Phys. Res. B 196, 194 (2002).

${ }^{12}$ B. Ravel and M. Newville, J. Synchrotron Radiat. 12, 537 (2005).

${ }^{13}$ J. J. Rehr, J. Mustre de Leon, S. I. Zabinsky, and R. C. Albers, J. Am. Chem. Soc. 113, 5135 (1991).

${ }^{14}$ J. Rouquette, J. Haines, V. Bornand, Ph. Papet, R. Astier, J. M. Leger, and F. Gorelli, Phys. Rev. B 65, 214102 (2002).

${ }^{15}$ Y. Shiratori, A. Magrez, and C. Pithan, J. Eur. Ceram. Soc. 25, 2075 (2005).

${ }^{16}$ N. Klein, E. Hollenstein, D. Damjanovic, H. J. Trodahl, N. Setter, and M. Kuball, J. Appl. Phys. 102, 014112 (2007). 